\title{
WI - Call for Papers Heft 5/2013
}

\section{Green IS - Informationssysteme für ökologische Nachhaltigkeit}

DOI 10.1007/s11576-011-0303-1

\author{
Die Autoren \\ Prof. Dr. Jan vom Brocke ( $\varangle)$ \\ Dr. Stefan Seidel \\ University of Liechtenstein \\ 9490 Vaduz \\ Liechtenstein \\ jan.vom.brocke@uni.li \\ stefan.seidel@uni.li
}

Prof. Dr. Peter Loos

Saarland University

66123 Saarbrücken

Deutschland

loos@iwi.uni-sb.de

Prof. Dr. Richard T. Watson

University of Georgia

Athens, GA 30602-6273

USA

rwatson@terry.uga.edu

Online publiziert: 2012-01-17

This article is also available in English via http://www.springerlink.com and http://www.bise-journal.org: vom Brocke J, Loos P, Seidel S, Watson RT (2012) BISE - Call for Papers, Issue 5/2013. Green IS - Information Systems for Environmental Sustainability. Bus Inf Syst Eng. doi: 10.1007/ s12599-011-0195-z.

C) Gabler Verlag 2012

\section{Schwerpunktthema}

Informations- und Kommunikationstechnologie wird oft als Verursacher eines gesteigerten Energieverbrauchs thematisiert. Unter dem Begriff Green IT werden daher Technologien entwickelt, die insbesondere auf eine möglichst stromsparende Informationsverarbeitung abzielen. Jüngere Studien belegen jedoch, dass gerade der intelligente Einsatz von Informationssystemen auch einen wichtigen Beitrag zur ökologischen Nachhaltigkeit leisten kann, der sowohl über Prozesse, als auch Produkte und Dienstleistungen wirkt: Informationssysteme tragen wesentlich zur Erhöhung der Ressourceneffizienz von Unternehmen bei, erlauben durch Virtualisierung und Delokalisierung ökologisch nachhaltige Arbeitsprozesse, haben Einfluss auf unser individuelles Verständnis und Verhalten in Bezug auf Nachhaltigkeit und ermöglichen es Organisationen Compliance-Anforderungen und sozialen Normen besser zu entsprechen. In diesem Zusammenhang sind Konzepte wie Energieinformatik (Energy Informatics) und Green Business Process Management (Green BPM) entstanden. Die Wirtschaftsinformatik ist gefordert - hat aber auch eine große Chance - zu untersuchen, welchen Beitrag Informationssysteme zur ökologischen Nachhaltigkeit von Wirtschaft und Gesellschaft leisten können.

Dieses Schwerpunktheft lädt zur Einreichung rigoroser Studien verschiedener Forschungsdesigns ein. Der Fokus liegt auf lösungsorientierten Studien, die zeigen, wie die Innovationskraft von Informationssystemen genutzt werden kann, um einen Beitrag zur Steigerung der ökologischen Nachhaltigkeit, sowohl auf individueller, organisationaler und gesellschaftlicher Ebene zu leisten. Willkommen sind sowohl Beiträge zur Entwicklung und Überprüfung von Theorie als auch zur Gestaltung und Evaluation von Artefakten, die deutlich machen, dass sie ökologisch nachhaltiges Handeln fördern können. Eine besondere Herausforderung sehen wir darin, aufzuzeigen, wie sich der Einsatz von IT für nachhaltigkeitsbezogenes Handeln vom Einsatz in "konventionellen“ Bereichen der Wirtschaftsinformatikforschung unterscheidet.

Beiträge aus Forschung und Praxis sind u. a. zu folgenden (gerne auch weiteren) Themenfeldern erwünscht:
- Gestaltung von Informationssystemen für nachhaltige Prozesse

- IT-gestützte Nachhaltigkeitstransformation

- Anwendung von Theorien der Wirtschaftsinformatik auf nachhaltigkeitsbezogene Themen

- Forschungsmethoden für die Untersuchung nachhaltigkeitsbezogener Themen der Wirtschaftsinformatik

- Einsatzszenarien von Informationssystemen für ökologische Nachhaltigkeit

- Supply Chain Management und Nachhaltigkeit

- Business Intelligence und Performance Measurement für Nachhaltigkeitsmanagement

- Systemorientierte Ansätze für IT-gestützte ökologische Nachhaltigkeit

- Kennzahlen- und Berichtssysteme für das Nachhaltigkeitsmanagement

- Energieinformatik

- Green Business Process Management

\section{Einreichung von Beiträgen}

Bitte reichen Sie Beiträge für die $\mathrm{Ru}$ briken WI - Aufsatz und WI - State of the Art bis spätestens 2012-11-01 über das Online-Begutachtungssystem (http://www.editorialmanager.com/buis/) ein. Bitte beachten Sie die Hinweise $\mathrm{zu}$ formaler Gestaltung und Umfang von Beiträgen für die WIRTSCHAFTSINFORMATIK/Business \& Information Systems Engineering (BISE). Vollständige Beiträge sollten höchstens 50.000 Zeichen einschließlich Leerzeichen umfassen, abzüglich 5.000 Zeichen je Seite an Bildern. Ausführliche Autorenrichtlinien stehen unter http://www.wirtschaftsinformatik.de zum Download bereit.

Eingereichte Beiträge werden anonymisiert von mehreren Gutachtern in einem doppelt-blinden Verfahren auf Relevanz, Originalität und fachliche Qualität beurteilt. Neben den Herausgebern des 
Schwerpunktthemas und jenen der Zeitschrift wirken dabei weitere ausgewiesene internationale Persönlichkeiten aus Wissenschaft und Praxis mit.

Ergänzend $\mathrm{zu}$ den Aufsätzen sind auch weitere Einreichungen zum Schwerpunktthema willkommen.

Angenommene Beiträge erscheinen identisch in Deutsch und Englisch. Die deutschsprachige Fassung erscheint in
WIRTSCHAFTSINFORMATIK, die englischsprachige in Business \& Information Systems Engineering (BISE).

\section{Zeitplan}

Einreichung von Beiträgen: 2012-11-01 Benachrichtigung der Autoren: 2012-12-27
Abschluss der ersten Überarbeitung: 2013-02-28

Benachrichtigung der Autoren: 2013-04-18

Ggf. Abschluss einer zweiten Überarbeitung (einsprachig): 2013-05-23

Ggf. Abschluss einer zweiten Überarbeitung (zweisprachig): 2013-06-20

Geplanter Erscheinungstermin Heft 5/2013: Oktober 2013 